\title{
Coordination of the in situ payload of Solar Orbiter
}

A. P. Walsh ${ }^{1}$, T. S. Horbury ${ }^{2}$, M. Maksimovic ${ }^{3}$, C. J. Owen ${ }^{4}$, J. Rodríguez-Pacheco ${ }^{5}$, R. F. Wimmer-Schweingruber ${ }^{6}$, I. Zouganelis ${ }^{1}$, C. Anekallu ${ }^{4}$, X. Bonnin ${ }^{5}$, R. Bruno ${ }^{7}$, I. Carrasco Blázquez ${ }^{2}$, I. Cernuda ${ }^{5}$, T. Chust ${ }^{8}$, A. De Groof ${ }^{1}$, F. Espinosa Lara ${ }^{5}$, A. N. Fazakerley ${ }^{4}$, H. R. Gilbert ${ }^{9}$, R. Gómez-Herrero ${ }^{5}$, G. C. Ho ${ }^{10}$, S. Krucker ${ }^{11}$, S. T. Lepri ${ }^{12}$, G. R. Lewis ${ }^{4}$, S. Livi ${ }^{13}$, P. Louarn ${ }^{14}$, D. Müller ${ }^{15}$, T. Nieves-Chinchilla ${ }^{9}$, H. O’Brien ${ }^{2}$, P. Osuna ${ }^{1}$, P. Plasson ${ }^{3}$, J. M. Raines ${ }^{12}$, A. P. Rouillard ${ }^{14}$, O. C. St. Cyr ${ }^{9}$, L. Sánchez ${ }^{1}$, J. Soucek ${ }^{16}$, A. Varsani ${ }^{4,17}$, D. Verscharen ${ }^{4,18}$, C. J. Watson ${ }^{1}$, G. Watson ${ }^{4}$, and D. R. Williams ${ }^{1}$

${ }^{1}$ European Space Agency, ESAC, Camino Bajo Del Castillo, s/n, 28692 Villanueva de la Cañada, Madrid, Spain e-mail: andrew.walsh@esa.int

2 Department of Physics, Imperial College London, London SW7 2AZ, UK

3 LESIA, Observatoire de Paris, Meudon, France

${ }^{4}$ Department of Space and Climate Physics, University College London, Mullard Space Science Laboratory, Holmbury St., Mary RH5 6NT, UK

${ }^{5}$ Universidad de Alcalá, Space Research Group, 28805 Alcalá de Henares, Spain

${ }^{6}$ Institut für Experimentelle und Angewandte Physik, Christian-Albrechts-Universität zu Kiel, Kiel, Germany

7 IAPS-INAF, via Fosso del Cavaliere 100, 00133 Roma, Italy

8 LPP, CNRS, Ecole Polytechnique, Sorbonne Université, Observatoire de Paris, Université Paris-Saclay, PSL Research University, Palaiseau, Paris, France

9 NASA Goddard Space Flight Center, Greenbelt, MD, USA

10 Johns Hopkins University Applied Physics Laboratory, Laurel, MD, USA

11 University of Applied Sciences and Arts Northwestern Switzerland, 5210 Windisch, Switzerland

12 Department of Climate and Space Sciences and Engineering, University of Michigan, Ann Arbor, Michigan, USA

13 Southwest Research Institute, San Antonio, TX, USA

14 IRAP, Université de Toulouse, CNRS, CNES, UPS, Toulouse, France

15 European Space Agency, ESTEC, Keplerlaan 1, 2201 AZ Noordwijk, The Netherlands

16 Institute of Atmospheric Physics, CAS, Prague, Czechia

17 Space Research Institute, Austrian Academy of Sciences, Graz, Austria

18 Space Science Center, University of New Hampshire, Durham, NH 03824, USA

Received 11 October 2019 / Accepted 8 December 2019

\section{ABSTRACT}

\begin{abstract}
Solar Orbiter's in situ coordination working group met frequently during the development of the mission with the goal of ensuring that its in situ payload has the necessary level of coordination to maximise science return. Here we present the results of that work, namely how the design of each of the in situ instruments (EPD, MAG, RPW, SWA) was guided by the need for coordination, the importance of time synchronisation, and how science operations will be conducted in a coordinated way. We discuss the mechanisms by which instrument sampling schemes are aligned such that complementary measurements will be made simultaneously by different instruments, and how burst modes are scheduled to allow a maximum overlap of burst intervals between the four instruments (telemetry constraints mean different instruments can spend different amounts of time in burst mode). We also explain how onboard autonomy, inter-instrument communication, and selective data downlink will be used to maximise the number of transient events that will be studied using high-resolution modes of all the instruments. Finally, we briefly address coordination between Solar Orbiter's in situ payload and other missions.
\end{abstract}

Key words. space vehicles: instruments - solar wind - Sun: general - Sun: particle emission - Sun: radio radiation

\section{Introduction}

Coordination will be the key to the scientific success of Solar Orbiter, the next solar physics mission of the European Space Agency (ESA) jointly developed with NASA (Müller et al. 2020). This is reflected in the science objectives of the mission, the capabilities of the platform and the payload, and has driven the design of the operations concept. Observations will need to be coordinated between the remote sensing and in situ payload, amongst the remote sensing instruments themselves and also amongst the in situ instruments. The science return of Solar Orbiter and other contemporary space missions, particularly Parker Solar Probe (Fox et al. 2016) and Bepi Colombo (Benkhoff et al. 2010), and ground-based facilities like the Daniel K. Inouye Solar Telescope (see Tritschler et al. 2016, for example) will also be enhanced through cross-facility coordination, as far as is possible within the constraints under which each facility operates.

This paper focuses on the work of Solar Orbiter's in situ coordination working group, and presents the rationale behind and requirements for coordination between the in situ instruments 
aboard the Solar Orbiter spacecraft, such that synchronisation of sampling, real-time transfer of data, and coordinated burstmode triggering will maximise the scientific usefulness of Solar Orbiter's unique in situ dataset. It describes the data shared between the in situ instruments onboard, the low-latency data products that will be returned by them, and how each of these are planned to be used in science operations.

This represents only a small fraction of the coordination that will be needed to make Solar Orbiter a success. Coordination between the remote sensing instruments is described by Auchère et al. (2020); how coordinated observations are required to address Solar Orbiter's detailed science objectives, and how the operations concept has been designed to make this possible are discussed by Zouganelis et al. (2020) and Sánchez et al. (in prep.), respectively. Finally, how models and simulations will contribute to Solar Orbiter's coordinated science is discussed by Rouillard et al. (2020).

Solar Orbiter's in situ payload consists of four instruments: the Energetic Particle Detector (EPD; Rodriguez-Pacheco et al. 2020), the Magnetometer (MAG; Horbury et al. 2020), the Radio and Plasma Waves Instrument (RPW; Maksimovic et al. 2020), and finally the Solar Wind Analyser (SWA; Owen et al. 2020). Each instrument is described in detail in the respective instrument paper, but key characteristics are summarised here for convenience.

The EPD is a suite of sensors dedicated to measuring electrons, protons, and heavy ions with energies from a few $\mathrm{keV}$ to tens of $\mathrm{MeV}$ for electrons and a few $\mathrm{keV}$ or $\mathrm{keV} /$ nuc to hundreds of $\mathrm{MeV}$ or $\mathrm{MeV} / \mathrm{nuc}$ for protons or heavier ions. It consists of four sensors: the SupraThermal Electron and Proton (STEP) sensor, the Electron Proton Telescope (EPT), the High Energy Telescope (HET), and the Suprathermal Ion Spectrograph (SIS). All four of the EPD sensors have a field-of-view looking sunward along a nominal Parker Spiral (Parker 1958) direction for typical solar wind speeds and heliocentric distances. Additionally, EPT and HET have second fields of view looking anti-sunward along the Parker spiral direction, and EPT, HET, and SIS also have other fields of view examining intermediate pitch angles. Furthermore, STEP's detector is divided into pixels that provide directional information within its field of view.

The MAG consists of two tri-axial fluxgate sensors, positioned approximately $1.2 \mathrm{~m}$ and $3.5 \mathrm{~m}$ along the spacecraft instrument boom, which extends behind the main spacecraft bus. The MAG can sample the DC magnetic field at up to 16 vectors per second in normal mode and 128 vectors per second in burst mode.

The RPW is a comprehensive radio and plasma waves suite, providing in situ measurements of electric and magnetic fields and waves from "dc" to a few hundred $\mathrm{kHz}$. It also measures solar radio emissions at frequencies of up to $16 \mathrm{MHz}$. The RPW is composed of three electric monopole antennae of $\sim 6 \mathrm{~m}$ in length and a search coil magnetometer with three axes sensitive to fluctuations with frequencies of $\leq 10 \mathrm{kHz}$ and a single axis sensitive to frequencies between $10 \mathrm{kHz}$ and $500 \mathrm{kHz}$. These sensors feed into three different receivers, the time domain sampler (TDS), the Low Frequency Receiver (LFR), and the Thermal Noise Receiver/High Frequency Receiver (TNR/HFR). The RPW also has a unit to bias the electric antennae and provide measurements of spacecraft potential. Its data products include waveform measurements of various frequencies and durations, depending on operating mode, and electric and magnetic wave power spectra and spectral matrices.

The SWA consists of three sensors and a data processing unit. The Electron Analyser System (EAS) provides 3D electron velocity distribution functions with almost $4 \pi$ steradian coverage
In Situ Fields-of-View

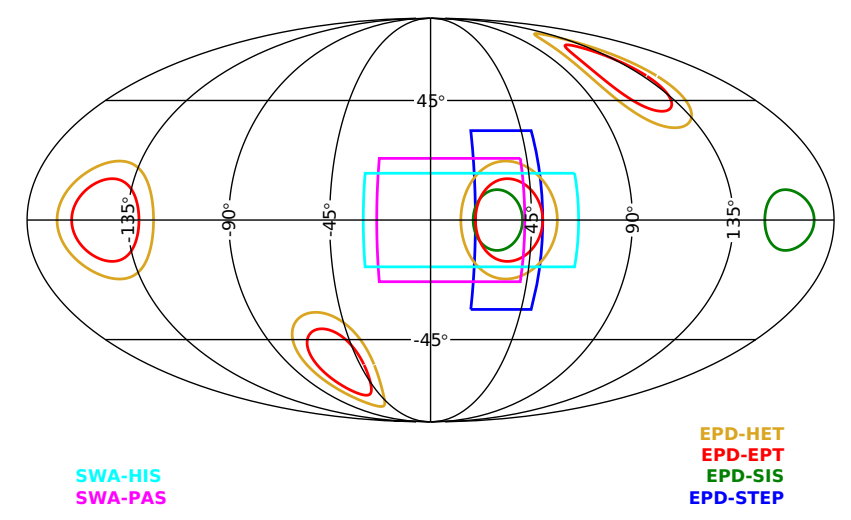

Fig. 1. Fields of view of SWA-HIS, SWA-PAS, and all EPD sensors. SWA-EAS samples the full sky except for some blockages due to the spacecraft and its appendages near $(0,0)$, i.e. Sunward.

between the energies of $0.5 \mathrm{eV}$ and $5 \mathrm{keV}$ every $100 \mathrm{~s}$, as well as moments of those distribution functions every $4 \mathrm{~s}$ and $8 \mathrm{~Hz}$ 2D pitch angle snapshots in burst mode. The Heavy Ion Sensor (HIS) provides heavy ion spectra with independent mass, energy, and charge discrimination at cadences of $30 \mathrm{~s}$ in burst mode and $300 \mathrm{~s}$ otherwise. Finally, the Proton Alpha Sensor (PAS) provides $3 \mathrm{D}$ VDFs of protons and alpha particles every $4 \mathrm{~s}$ and also higher time-resolution snapshots every $5 \mathrm{~min}$. The Data Processing Unit (DPU) is the only SWA data interface with the S/C. It is in charge of supporting EAS and PAS with power, functionality control, temporary storage, data compression, communication, and computational capability, and routes data packets between HIS and the S/C; HIS manages its own data handling and is powered directly from the spacecraft. Both HIS and PAS have fields of view that have been designed to observe the solar wind direction with enough margin to take into account the different aberration angles caused by Solar Orbiter's varying tangential velocity along its eccentric orbit.

The fields of view of HIS, PAS, and the EPD sensors are plotted on a Mollweide projection of the sky in Fig. 1, with Sunward at $\left(0^{\circ}, 0^{\circ}\right)$.

\section{Coordination by design}

Missions whose objectives include the study of space plasmas have always required coordination between the various payload elements, no matter the context (e.g. Wenzel et al. 1992; Escoubet et al. 2001), and Solar Orbiter is no exception. While coordination of operations is important, a payload that is designed to make coordinated, complementary measurements is necessary. Wherever possible, Solar Orbiter's in situ payload has been designed to do that.

In terms of particle measurements, the energy ranges of SWA (-EAS and -PAS, for electrons and protons, respectively) and EPD-STEP are designed to overlap allowing the possibility to construct an electron spectrum from a few eV to a few hundred $\mathrm{keV}$, provided sufficiently high particle fluxes. Similarly, for some species of heavier ions, SWA-HIS and EPD-SIS also overlap in energy range, ensuring the suprathermal ion population can be well characterised.

In terms of field measurements, MAG and RPW have an overlapping frequency range such that in some operating modes a power spectrum of the magnetic field can be constructed from 
$\sim \mathrm{mHz}$ to $\sim \mathrm{kHz}$ (see Kiyani et al. 2015, for example), and indeed the production of a merged data product is planned.

Furthermore, it is expected that closer to the Sun, quasithermal noise spectroscopy (e.g. Meyer-Vernet 1979; Zouganelis 2008) can be used to derive an absolute measurement of electron density and temperature with RPW, which can then be compared with the SWA measurements in order to improve the quality of data from both instruments.

\section{Coordination of operations}

Although some of the requirements for coordinated observation by Solar Orbiter's in situ payload are met simply by the characteristics of the various payload elements, some aspects of coordination require that the instruments be operated in a certain way. This section describes how the in situ payload will be operated to maximise the degree of coordination between the instruments.

\subsection{Normal and burst modes}

Each of Solar Orbiter's in situ instruments has a broad range of configuration settings that can alter the details of the measurements being made and therefore the resulting data products. Most of these are beyond the scope of this paper. However each instrument has defined normal and burst modes that are designed to address different science questions and need to be treated differently in terms of coordination.

The normal mode of each instrument, which will be operating the vast majority of the time, will be used to address the question of connectivity between the local solar wind and the Sun, and will provide sufficient information to study the solar wind at length scales larger than the kinetic particle scales. This does not require explicit coordination amongst the payload since the data products have been designed to be complementary.

The investigation of the small-scale or high-frequency kinetic physics of the solar wind, heliosphere, and transient structures therein, such as for example interplanetary shocks and type III radio bursts, require higher-resolution or more detailed data that cannot be returned all of the time within the telemetry constraints of the mission. This problem is addressed in two ways: first, through a scheme of regular, high-resolution, "snapshots" taken as part of normal mode; and second, through the definition of burst modes which take higher-resolution data, but at a low and variable duty cycle. Burst modes can further be considered as either scheduled or triggered, depending on whether the burst mode is entered via ground command or autonomously. Each of these three solutions implies a different level of coordination. The coordination of the normal mode snapshots is described in Sect. 3.2, while the planning of scheduled bursts is described in Sect. 3.3. Triggered burst modes, and their consequences for coordination, are addressed in Sect. 6, which describes onboard autonomy as applied to the in situ payload in general.

\subsection{Coordinated "Snapshot" sampling in normal mode}

The in situ payload has been designed to take coordinated datasets with as little intervention as possible by operations teams. The sampling schemes of instruments have been aligned such that where instruments return various parameters and data products with different cadences as part of their normal mode operations, the sampling of those parameters that enable more coordinated science is carried out in phase. Typically this is based on a five-minute cycle and is illustrated in Fig. 2.

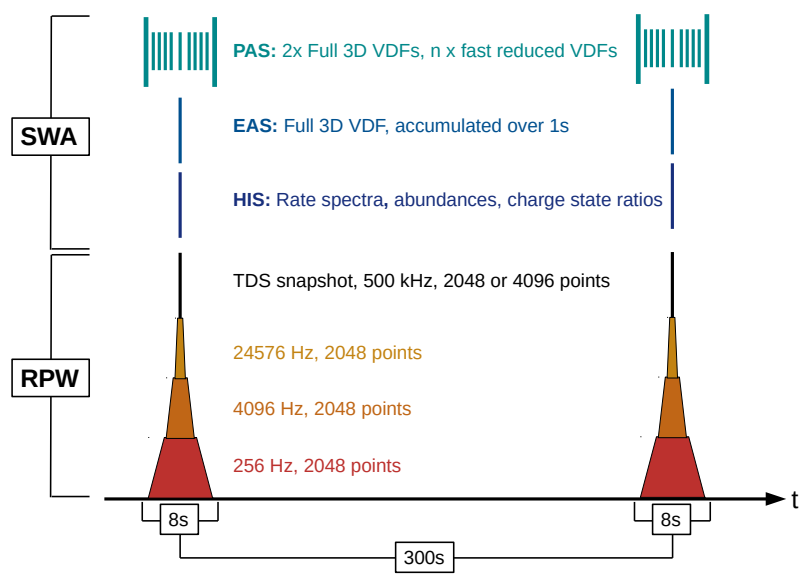

Fig. 2. Those RPW and SWA data that form part of the coordinated sampling scheme. Other data products and those that fall outside the $300 \mathrm{~s}$ cycle (e.g. EAS VDFs measured at $100 \mathrm{~s}$ and $200 \mathrm{~s}$ ) are not shown. We note that the PAS contribution is configurable and details may change in flight.

The driving measurements in the coordinated sampling scheme are the RPW waveform snapshots - waveforms containing 2048 or 4096 data points are measured in four frequency bands ranging from $256 \mathrm{~Hz}$ to $500 \mathrm{kHz}$. These are measured during an eight-second interval of snapshots every $300 \mathrm{~s}$. Over the same eight seconds, SWA-PAS also samples the proton and alpha velocity distribution functions at a higher cadence than usual, albeit over a reduced energy and angle range that is selected through a combination of instrument settings and an onboard identification of the peak of the proton VDF made immediately prior to the snapshot. The exact cadence of the VDF measurements can be tuned via instrument commanding, although higher cadences imply reduced angle and energy coverage or resolution. This coordinated sampling scheme will allow for more detailed measurements of wave-particle interactions at ion scales than would otherwise be possible. Similarly, SWAHIS data are produced at a cadence of $300 \mathrm{~s}$ and should be aligned with the PAS and RPW snapshots, while SWA-EAS 3D VDFs, sampled over $1 \mathrm{~s}$ at a cadence of $100 \mathrm{~s}$ will also be phased to provide the measurement at the centre of the snapshot interval.

In order for the sampling scheme to be successful, careful instrument commanding will be necessary to start the measurement cycle of each instrument such that the snapshots are aligned. This is one of the goals of the coordinated science planning that will be managed by the Science Operations Centre (SOC; Sánchez et al., in prep.).

\subsection{Coordinated science planning}

Given the agreed mission level science plan (Zouganelis et al. 2020), perhaps the most important decision in the context of in situ coordination that will be made during the long-term science planning process (where details of coordination are decided; Sánchez et al., in prep.) will be the frequency and times of coordinated burst modes.

After SOC has analysed the available telemetry and the SWT have set the science priorities for a given planning period, the amount of burst mode data that can be generated in that period by each instrument is known. This then sets the number of coordinated burst mode intervals that can be scheduled in that planning period. A coordinated burst mode interval is defined as an interval during which each in situ instrument team commits to 


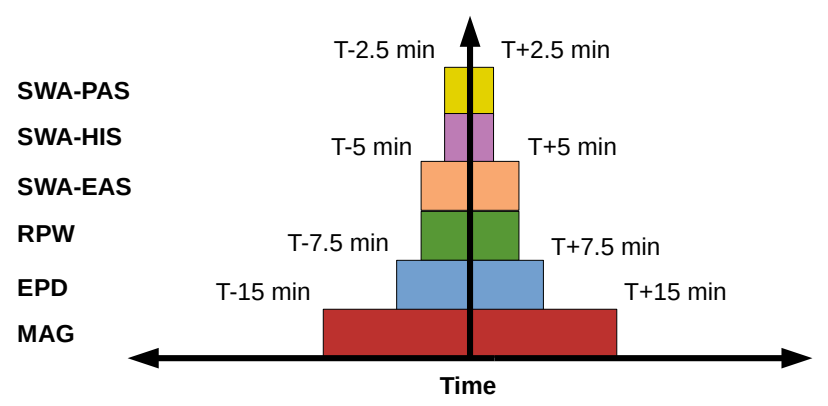

Fig. 3. Example coordinated burst illustrating the different amounts of time each instrument or sensor is in burst mode. Actual durations may vary depending on operational constraints, but all should be centred on the agreed burst mode time.

commanding their instrument to enter burst mode. The times of these intervals are decided by the Science Operations Working Group and communicated to the instrument teams in the formal planning products that are issued by the SOC (Sánchez et al., in prep.) for that planning period. We note that it may not always be the case that all the elements of an instrument take part in a given coordinated burst. The higher-energy sensors of EPD, for example, would not enter burst mode when fluxes were below a certain threshold and they were only measuring noise or the cosmic ray background. Since the proportion of telemetry available for burst mode is different for each instrument, for a given coordinated burst interval, the different instruments will necessarily be in burst mode for different durations. The time of the coordinated burst mode interval is therefore defined as the centre of the interval, such that all four instruments will be in burst mode for the entire duration of the shortest burst mode (typically that of SWA), with longer burst durations extending backwards and forwards in time. These nested burst mode durations are illustrated in Fig. 3, which gives an example of a coordinated burst mode with a duration such that one interval can be scheduled per day assuming nominal data allocations to each instrument. In practice, during some periods it will be possible to schedule more burst mode intervals than one per day, while in others, lower telemetry rates will mean fewer can be scheduled. It is also possible that an individual instrument or sensor will enter burst mode for a variable period of time for different coordinated bursts, depending on available telemetry, but the burst mode should always be centred on the agreed interval time.

An additional consideration in planning coordinated burst modes is whether or not the science goals of Solar Orbiter are best-served by aiming to produce, over the mission as a whole, a burst mode dataset that has equal coverage in time (e.g. one coordinated burst mode per day), or one that has equal coverage in heliocentric distance. At the time of writing, the best strategy has not yet been decided.

\section{Time synchronisation}

Solar Orbiter carries two sensors which measure the magnetic field: the MAG fluxgate and the RPW search coil, SCM. Fluxgates suffer from white noise but respond to a wide frequency range. Search coils on a three-axis stabilised spacecraft are not sensitive to very low frequencies, but have better high-frequency response. In practice, search coils outperform fluxgates at frequencies above a few Hertz. Low-pass filtered MAG and highpass filtered RPW-SCM measurements can be combined to produce a time series that offers the optimum response over all measured frequencies; this has been performed with other missions, such as Cluster and Magnetospheric Multiscale (MMS). Such a procedure requires accurate timing knowledge of the signals from both sensors to a precision of the order of $1 \mathrm{~ms}$.

Both RPW and MAG timestamp their data based on individual instrument clocks which are synchronised to spacecraft time using synchronisation packets on the SpaceWire data link. Each instrument has its own processing chain, including filters, which means that the timestamp on a vector is not precisely the time at which it was measured. A precise analytical determination of this discrepancy is challenging. In order to quantify the relative time variations between MAG and SCM timestamps, a test will be performed using the Solar Orbiter "flat sat" electrical testbench, with flight-representative development models of MAG and SCM. A coil will be used to generate a series of magnetic field signals at a set of frequencies; the resulting MAG and SCM data will be analysed to determine the potentially frequencydependent time delays between the two data sets. The results will be used to optimise the resulting merged magnetic field data set, which is expected to be produced for selected intervals of flight data during times of interest. The sampling frequency of the RPW waveform snapshots $(256 \mathrm{~Hz}, 4096 \mathrm{~Hz}, 24576 \mathrm{~Hz})$ are integer multiples of the MAG magnetic field cadence (16 vectors per second in Normal mode; usually 64 vectors per second in burst mode).

We note that the cadence of the particle measurements is considered slow enough relative to the fields measurements that detailed synchronisation activities beyond the phasing of the sampling schemes described in Sect. 3.2 are not needed. This is also true for the $8 \mathrm{~Hz}$ EAS pitch angle distribution products, which will almost always be collected in parallel with MAG burst mode.

\section{Instrument alignment}

While the remote sensing instruments have stringent requirements on co-alignment (and knowledge of that alignment; Auchère et al. 2020), which have been verified via an extensive test campaign, the requirements of the in situ payload are more modest and their alignment, and co-alignment, will have to be verified by analysis in flight. For the boom-mounted sensors (both MAG sensors, RPW-SCM and SWA-EAS), this is partially because in the nominal case the instrument boom orientation with respect to the spacecraft will only be known to an accuracy of $\sim 1^{\circ}$ after deployment.

Through careful analysis of the data returned by EAS, MAG, and RPW-SCM, it ought to be possible to reconstruct the coalignment of the various sensors and, through comparison with EPD or SWA-PAS and SWA-HIS data, potentially even the alignment of the two sections of the instrument boom. In order to check the coalignment of EAS and the MAG sensors, the magnetic field direction measured by MAG will be compared with the EAS measurement of the solar wind strahl (Rosenbauer et al. 1977) direction. The strahl flux peaks parallel or antiparallel to the local magnetic field (e.g. Graham et al. 2017, and references therein). Similarly, the existence of the solar wind ion beam (e.g. Feldman et al. 1974; Alterman et al. 2018), also aligned to the magnetic field, will potentially allow the instrument boom alignment to be reconstructed, since SWA-PAS is mounted on the main body of the spacecraft. Considering MAG and RPWSCM data in only their common frequency range will allow the co-alignment of the search coil magnetometer, and each of the fluxgates. Indeed this will be necessary to produce any merged science data products, and knowledge of the co-alignment 
between the RPW SCM and electric antennae is necessary for the characterisation of any electromagnetic fluctuations.

All of the potential alignment reconstructions described above depend on the availability of a large amount of data from well-calibrated individual instruments, and if they are carried out will result in an improvement in the quality of the science data later in the mission.

\section{Onboard autonomy and inter-instrument communication}

As described in Sect. 3, low-duty-cycle, high-resolution burst mode data are required to study small-scale kinetic physics with Solar Orbiter. The low duty cycle lowers the probability of the spacecraft encountering a transient structure in the solar wind, for example an interplanetary shock, while the instruments are in a scheduled burst mode. Furthermore, there is insufficient storage on board to constantly operate in burst mode and subsequently select interesting events on ground for downlink, as is done by the MMS mission for example (Burch et al. 2016; Baker et al. 2016). Instead, instruments continuously record their burst mode data stream in small rolling buffers that can typically store $\leq 10-15 \mathrm{~min}$ of observations. The contents of these buffers are then released to the spacecraft memory for downlink when an interesting event is detected by triggering algorithms running onboard. This is especially important considering there will be no real-time ground commanding of the payload during nominal science operations.

Two of the in situ instruments, EPD and RPW, have triggering algorithms. The EPD instrument triggers when the energetic particle fluxes in different energy ranges reach certain thresholds, directing the corresponding burst mode data streams from its individual sensors to the spacecraft mass memory. The EPD burst mode data are intended to sample the onset of energetic particle events at a higher cadence and energy resolution than would otherwise be possible. A more complicated triggering scheme is employed by RPW, which activates different burst mode data streams known as SBM1 and SBM2 (survey burst modes 1 and 2). Here, SBM1 is dedicated to understanding the physics of interplanetary shocks while SBM2 is tuned to study type III radio bursts. Although the event detection algorithms that decide when to activate SBM1 or SBM2 run on the RPW DPU (details of the detection algorithms are provided by Maksimovic et al. 2020, and references therein), they both require data products from several instruments: The SBM1 algorithm requires the magnetic field magnitude from $M A G$, and the solar wind density and velocity from SWA; the SBM2 algorithm requires electron flux from EPD. To meet this requirement, the Solar Orbiter spacecraft has the capability to share data, including platform housekeeping parameters and indeed burst mode triggers raised by RPW, amongst the payload. This is known as inter-instrument communication (IIC) and as well as allowing RPW to detect events, it enables EPD, MAG, and SWA to autonomously enter burst mode, that is, to transfer the contents of their rolling buffers to the spacecraft mass memory, in response to RPW's detection of those events. This provides the means for coordinated autonomous behaviour onboard and avoids the need for each instrument to implement its own shockdetection algorithm. This is particularly advantageous as the different instruments would not necessarily always detect the same shocks.

The IIC is managed by the onboard computer (OBC), which receives a housekeeping packet of agreed structure from each instrument, typically every second or eight times per second. The
Table 1. Selected parameters included in the inter-instrument communication packet.

\begin{tabular}{ll}
\hline \hline Source & Parameter \\
\hline Platform & Thruster firing flag \\
& 4 reaction wheel speeds \\
& 4 reaction wheel speed validities \\
\hline EPD & Data validity flag $(\times 8)$ \\
& 40 keV electron flux \\
& 60 keV electron flux \\
& 100 keV electron flux \\
& 200 keV electron flux \\
& 500 keV proton flux \\
& 8 burst mode flags \\
\hline MAG & B $($ Cartesian, SC coordinates $)$ \\
& Primary sensor range \\
& Operating mode \\
& Trigger source \\
& Last burst trigger time \\
\hline RPW & Spacecraft potential status flag \\
& SBM1 detection flag \\
& SBM2 detection flags \\
& Spacecraft potential \\
& SBM1 quality factor \\
& SBM1 trigger time \\
& SBM2 quality factor \\
& SBM2 trigger time \\
\hline SWA & Proton density \\
& Solar wind velocity vector \\
\hline &
\end{tabular}

OBC extracts parameters from each housekeeping packet, and places them in an onboard data pool. The parameters supplied by the instruments are subsequently extracted from the data pool and, along with relevant platform parameters, placed in the interinstrument communication packet, which is then distributed to each instrument, again with either a frequency of one per second or eight per second, depending on the needs of that instrument. The parameters included in the IIC packet and that are used by the in situ payload are listed in Table 1 . A simplified view (i.e. the loop through the OBC for payload parameters is not shown) of which parameters are used by each in situ instrument is plotted in Fig. 4. As well as the parameters listed in Table 1, each instrument includes the coarse onboard time (i.e. $\sim 1 \mathrm{~s}$ precision) so that other instruments can distinguish between recent, valid parameters and those left in the data pool after an instrument has been switched off, for example. The IIC via the OBC has the advantage of requiring less harness than dedicated interinstrument links and therefore represents mass and AIT (assembly, integration, and test) effort savings, although it does have the disadvantage of introducing some latency in data distribution.

In addition to providing the necessary information for burst mode triggering algorithms, IIC allows the in situ payload to use the data of the various instruments for other purposes. For example, SWA-EAS uses the magnetic field vector from MAG in order to electrostatically steer the sensors in burst mode so that its 2D burst mode product is comprised of a complete pitch angle distribution. Furthermore, SWA makes use of the spacecraft potential measurement from RPW in the calculation of onboard moments of the electron distribution function. The reaction-wheel speeds provided by the platform are used by RPW to choose which frequencies to notch in order to minimise contamination of the science data by noise from the reaction 


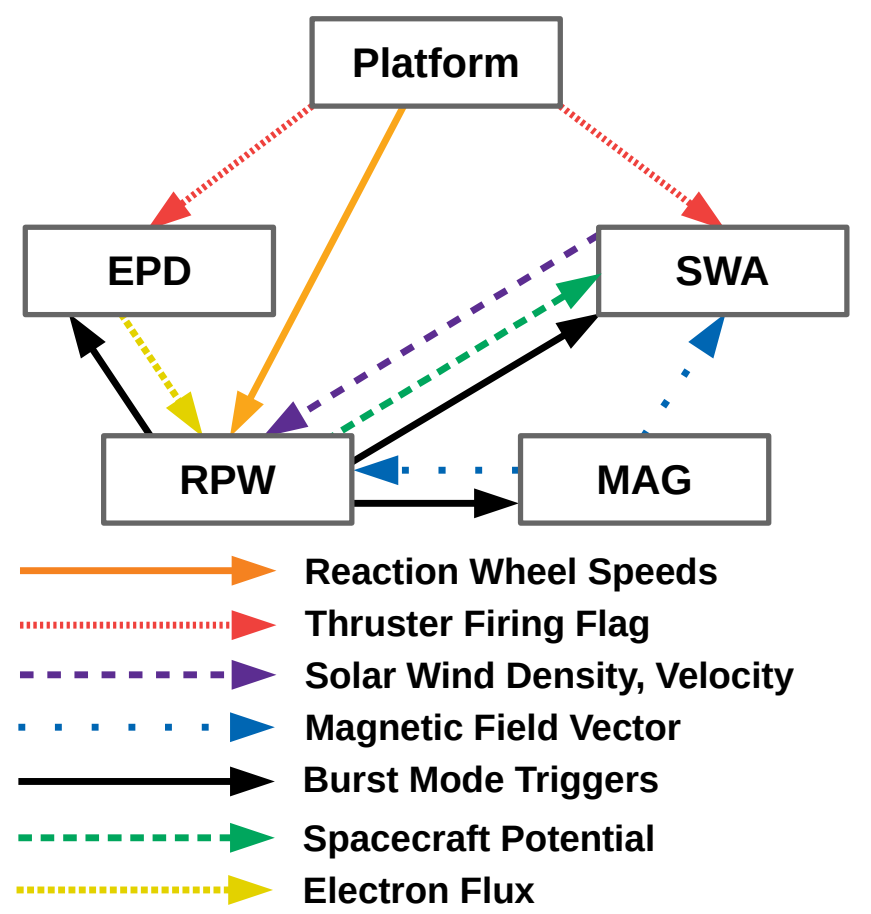

Fig. 4. Parameters exchanged via IIC that trigger a response from the receiving instrument. We note that all parameters pass through the spacecraft computer, but these interactions are omitted for clarity.

wheels. Remote sensing use of IIC is discussed by Auchère et al. (2020).

Autonomous onboard use of payload data relies heavily on accurate calibration of instruments and their data. Inaccurate calibrations will adversely affect measurement capabilities of a number of instruments that rely on parameters from other instruments to guide their observations. If the onboard MAG calibration is inaccurate then EAS will not sample accurate pitchangle distributions with sufficient coverage in the directions perpendicular and parallel to the magnetic-field vector. If PAS geometric factors are inaccurate, then the error in the onboard moment calculations will introduce inaccuracies in the SBM1 (shock) trigger. While some instruments require onboard knowledge of calibration to construct some of their own data products, that is not universally true. The requirement for IIC has influenced the design of MAG for example, which would not otherwise need to store and use calibration information onboard. In practice, updated calibration information is expected to be uploaded approximately monthly depending on the rate at which spacecraft-generated offsets change in flight.

As well as offering increased science performance, the IIC packet allows the spacecraft to distribute important safety information to the payload. For example, this packet contains a flag warning instruments of imminent thruster firings, which may occur autonomously. Enhanced pressure during these intervals could damage SWA in particular. Despite the warning flags, the operations concept is such that the vast majority of thruster firings will be commanded from the ground, thus enabling instruments to prepare and enter safe mode as needed during these periods. Additionally, the presence or absence of the IIC packet itself is used to communicate spacecraft status to the instruments. If the distribution of the IIC packet stops, this is a signal to the instruments that the spacecraft is about to enter safe mode and that they should react accordingly.

\section{Selection of burst events and low-latency data}

Despite the presence of burst mode triggers, and even discounting the possibility of false triggers, the number of interplanetary shock, type III radio burst and energetic particle events that Solar Orbiter is predicted to observe will still generate more burst mode telemetry than can realistically be downlinked. The proportion of the data allocation of each instrument dedicated to burst mode is different, as is the mechanism by which each instrument manages that allocation. This means some coordination is required in managing the downlink of burst events to ensure the largest possible number of complete events (i.e. those with all the relevant data downlinked) is available.

Both RPW and EPD have chosen to make use of a selective downlink scheme, whereby they can choose a subset of triggered burst events for downlink based on data quality or scientific value. Scheduled bursts are excluded from this mechanism and are always downlinked. Conversely, SWA uses a bookkeeping algorithm that allows the instrument to autonomously trade scheduled burst modes against triggered burst modes, while protecting those scheduled bursts that have been agreed by the SOWG during mission planning (see Sect. 3). Of the in situ instruments, MAG is expected to be able to dedicate the highest proportion of its telemetry to burst mode, and therefore expects to always be able to enter burst mode in conjunction with any other instrument.

Due to the limitations of the onboard mass memory, RPW and EPD will have a finite window of opportunity to downlink a given burst event before it is overwritten by subsequent events. This window of opportunity will often be shorter than the latency of the normal mode data that was taken at the same time as the burst event, so the normal mode science data will not always be available to assess whether or not the burst event is of sufficient quality to downlink. To address this, a subset of science data - low-latency data - will be downlinked during every communications pass and all data will be made available immediately to each instrument team. The low-latency dataset (see Sánchez et al., in prep., for details), together with instrument housekeeping data will enable the EPD and RPW teams to assess the quality of any burst mode events, including for example whether or not SWA was able to respond to a trigger, and therefore choose the best events to downlink.

\section{Coordination with the remote sensing payload and other missions}

The coordination amongst the in situ payload described above is focussed on ensuring each instrument makes complementary measurements at the same time. Coordination between the in situ and remote sensing payloads is somewhat different purely because of the different timescales involved. The solar wind for example will take $\sim 1$ day to reach Solar Orbiter while the spacecraft is close to perihelion. Therefore, it is necessary to image the source region of the parcel of solar wind that will reach the location of the spacecraft after the solar wind propagation time has passed. Similarly, energetic particles tend to propagate along the interplanetary magnetic field, albeit with a sometimes large spread in longitude (e.g. Prise et al. 2014; Gómez-Herrero et al. 2015); imaging the footpoints of the magnetic field lines connected to the spacecraft is therefore necessary. These are two of the central challenges of science planning for Solar Orbiter that are addressed by Sánchez et al. (in prep.) and Zouganelis et al. (2020) and will require significant modelling support. Community efforts in this direction are described 
by Rouillard et al. (2020). The in situ instruments have been designed such that those data products that will be available whenever the payload is operating will provide sufficient in situ data to address the science goals involving establishing connectivity between the Sun and Solar Orbiter. Coordination in that context therefore amounts to careful choice of pointing and does not involve special operations on the part of the in situ payload. The low-latency parameters have also been selected to provide a way of establishing connectivity - ion composition information, for example - and can therefore be used to provide context to the choice of target for the high-resolution telescopes.

Similarly, coordination between Solar Orbiter's in situ payload and other missions is a matter of broader mission planning. For example, it may be advantageous to concentrate more burst mode intervals or increase the cadence of normal mode data during radial or magnetic conjunctions with Parker Solar Probe, or indeed for Solar Orbiter to act as an upstream monitor for Bepi Colombo at Mercury. In either case, a trade-off with other science priorities would be necessary and the decision would be made by the SWT.

\section{Summary}

The science return of Solar Orbiter will be greatly enhanced through coordination between its instruments. Here we summarise the ways in which the in situ payload (EPD, MAG, RPW and SWA) will work together to achieve that goal. Each payload has been designed, wherever possible, to be complementary with the others including overlapping energy ranges, frequency ranges, and fields of view.

The payload will be operated in as coordinated a manner as possible, with the sampling schemes of the different instruments in phase, enabling the investigation of solar wind kinetic physics in both normal and burst modes. Burst modes will often be commanded from the ground such that all instruments are in burst mode during the same intervals. Inter-instrument communication will allow RPW to autonomously detect interplanetary shocks and in situ type III radio bursts based on data from MAG, SWA, and EPD. The same mechanism will let RPW inform the rest of the payload of the detection and hence all four in situ instruments can respond by routing data from a rolling buffer to the spacecraft memory and also entering burst mode without ground intervention. The EPD instrument will also enter into burst mode autonomously in response to energetic particle events. This will enable Solar Orbiter to capture many more solar wind transients in detail than would otherwise be possible, given the telemetry constraints under which the mission will operate. Telemetry will be focussed on the most scientifically interesting transient events through the use of a selective downlink scheme by EPD and RPW, whereby a subset of the detected events can be chosen for downlink. This process is supported by a limited set of low-latency data that will give the instrument teams visibility of what was measured in (usually) the previous $24 \mathrm{~h}$.

A combined MAG-RPW/SCM magnetic field data product covering a wide frequency range will be produced, using grounddetermined relative time delays to optimise the merging process.

The data from the in situ payload can be used in a coordinated way not only to address the science goals of the mission, but also to aid in cross-calibration and characterisation of instrument and spacecraft boom alignment. This will indeed require careful statistical analysis of data, but if done will provide an improvement to the quality of the in situ data later in the mission.

Finally, Solar Orbiter's in situ payload will make a valuable contribution to campaigns conducted in coordination with other assets, notably Parker Solar Probe and Bepi Colombo. Although the normal mode data from Solar Orbiter, available all of the time, will be sufficient for coordinated studies of the large-scale evolution of the solar wind, where possible its in situ payload will be operated in such a way as to maximise the scientific return of these joint campaigns.

Acknowledgements. Javier Rodríguez-Pacheco acknowledges FEDER/MCIUAEI/Project ESP2017-88436-R. Roberto Bruno acknowledges funding by ASI under contract ACCORDO ATTUATIVO n. 2018-30-HH.O. Daniel Verscharen was supported by the STFC Ernest Rutherford Fellowship ST/P003826/1. The Solar Orbiter magnetometer team is supported by the UK Space Agency grant $\mathrm{ST} / \mathrm{P} 00217 \mathrm{X} / 1$. SWA is supported in the UK by UK Space Agency grant ST/T001356/1. M. Maksimovic thanks both CNES and CNRS for supporting the RPW instrument. Solar Orbiter is an ESA-led mission with strong NASA participation.

\section{References}

Alterman, B. L., Kasper, J. C., Stevens, M. L., \& Koval, A. 2018, ApJ, 864, 112

Auchère, F., Andretta, V., Antonucci, E., et al. 2020, A\&A, 642, A6 (Solar Orbiter SI)

Baker, D. N., Riesberg, L., Pankratz, C. K., et al. 2016, Space Sci. Rev., 199, 545

Benkhoff, J., van Casteren, J., Hayakawa, H., et al. 2010, Planet. Space Sci., 58, 2

Burch, J. L., Moore, T. E., Torbert, R. B., \& Giles, B. L. 2016, Space Sci. Rev., 199, 5

Escoubet, C. P., Fehringer, M., \& Goldstein, M. 2001, Ann. Geophys., 19, 1197

Feldman, W. C., Asbridge, J. R., Bame, S. J., \& Montgomery, M. D. 1974, Rev. Geophys. Space Phys., 12, 715

Fox, N. J., Velli, M. C., Bale, S. D., et al. 2016, Space Sci. Rev., 204, 7

Gómez-Herrero, R., Dresing, N., Klassen, A., et al. 2015, ApJ, 799, 55

Graham, G. A., Rae, I. J., Owen, C. J., et al. 2017, J. Geophys. Res. (Space Phys.), 122, 3858

Horbury, T. S., O’Brien, H., Carrasco Blazquez, I., et al. 2020, A\&A, 642, A9 (Solar Orbiter SI)

Kiyani, K. H., Osman, K. T., \& Chapman, S. C. 2015, Phil. Trans. R. Soc. London Ser. A, 373, 20140155

Maksimovic, M., Bale, S. D., Chust, T., et al. 2020, A\&A, 642, A12 (Solar Orbiter SI)

Meyer-Vernet, N. 1979, J. Geophys. Res., 84, 5373

Müller, D., St. Cyr, O. C., Zouganelis, I., et al. 2020, A\&A, 642, A1 (Solar Orbiter SI)

Owen, C. J., Bruno, R., Livi, S., et al. 2020, A\&A, 642, A16 (Solar Orbiter SI)

Parker, E. N. 1958, ApJ, 128, 664

Prise, A. J., Harra, L. K., Matthews, S. A., Long, D. M., \& Aylward, A. D. 2014, Sol. Phys., 289, 1731

Rodriguez-Pacheco, J., Wimmer-Schweingruber, R. F., Mason, G. M., et al. 2020, A\&A, 642, A7 (Solar Orbiter SI)

Rouillard, A. P., Pinto, R. F., Vourlidas, A., et al. 2020, A\&A, 642, A2 (Solar Orbiter SI)

Rosenbauer, H., Schwenn, R., Marsch, E., et al. 1977, J. Geophys., 42, 561

Tritschler, A., Rimmele, T. R., Berukoff, S., et al. 2016, Astron. Nachr., 337, 1064

Wenzel, K. P., Marsden, R. G., Page, D. E., \& Smith, E. J. 1992, A\&AS, 92, 207

Zouganelis, I. 2008, J. Geophys. Res.: Space Phys., 113

Zouganelis, I., De Groof, A., Walsh, A., et al. 2020, A\&A, 642, A3 (Solar Orbiter SI) 